\title{
Maestría en Tecnologías de la información aplicadas a la Educación
}

\author{
Ambientes virtuales de aprendizaje b-learning y su incidencia en la \\ motivación y estrategias de aprendizaje en Estudiantes de secundaria
}

\section{Autor: ARIAS AGUIRRE, Olga Milena}

Palabras claves: b-learning, estrategias de aprendizaje, motivación, MSLQ, orientación a las metas, valoración de la tarea, autoeficacia, control de aprendizaje, ansiedad, repaso, elaboración, organización, pensamiento crítico, autorregulación, manejo del tiempo, aprendizaje con pares, búsqueda de ayuda.

Fecha de elaboración del resumen: 23 de febrero de 2010

\section{Descripción}

Trabajo de grado para optar al título de Magister en Tecnologías de la Información Aplicadas a la Educación, que tiene como propósito fundamental, Identificar qué incidencia tienen los ambientes virtuales b-learning en la motivación y estrategias de aprendizaje en estudiantes de secundaria del Colegio Hacienda los Alcaparros

\section{Contenido}

El trabajo integra la justificación, los antecedentes, el marco teórico, los objetivos y la metodología para la realización de un estudio que determinó las incidencias que tienen los ambientes b-learning en la motivación y estrategias de aprendizaje en estudiantes de secundaria, este trabajo se hizo como tesis de grado para optar al título de magister en tecnologías de información aplicadas a la educación. El primer capítulo presenta la introducción, el segundo capítulo presenta la contextualización del problema, el tercer capítulo presenta estudios y planteamientos de diversos autores que anteceden este trabajo de investigación, destacando sus resultados y hallazgos que son el soporte sobre el cual se construyen los postulados validados en esta investigación. El cuarto capítulo presenta algunos conceptos y teorías sobre el aprendizaje bajo modalidad b-learning, las estrategias de aprendizaje, aspectos conceptuales sobre la motivación y el instrumento de evaluación MSLQ utilizado en esta investigación. El quinto capítulo presenta el diseño de la investigación que incorpora la definición del problema, hipótesis, población y muestra, instrumento, diseño del ambiente experimental, recolección y procesamiento de los datos y análisis de los datos. El capítulo sexto presenta las conclusiones producto del trabajo, y por último se relacionan las fuentes consultadas para la fundamentación de este trabajo de nvestigación.

\section{Metodología}

La metodología de investigación utilizada es de tipo cuasi experimental, la experimentación se desarrolló con dos grupos de estudiantes de secundaria: unos en ambiente presencial (clases tradicionales) y otros interactuando con ambientes virtuales de aprendizaje b-learning. Se realizó 
una prueba post-test a los grupos utilizando el cuestionario de autoinforme: Motivated Stretegies for Learning Questionnaire (MSLQ) de (Pintrich, 1991). El cuestionario consta de dos secciones: una referida a la motivación y la otra relativa al uso de estrategias de aprendizaje. Para realizar el estudio se desarrolló un ambiente b-learning, el cual tiene un sitio para el curso y para cada una de las asignaturas del curso, el profesor tiene un espacio para publicar a los estudiantes anuncios, mensajes, links, archivos, actividades, tareas y eventos. Los estudiantes pueden consultar las próximas actividades del curso, actividades realizadas anteriormente, la calificación, las notas del periodo y el avance en sus objetivos curriculares. Cada sitio tiene también la opción de contar con foros de discusión, wikis y blogs. Actualmente el Colegio Hacienda los Alcaparros brinda a los estudiantes de secundaria este ambiente virtual de aprendizaje "Myschool" como apoyo a sus clases tradicionales.

\section{Conclusiones}

De acuerdo con el análisis de resultados y de cara a los objetivos planteados en este estudio se llevó a cabo la consulta documental sobre ambientes B-learning, estrategias de aprendizaje, motivación y el instrumento de (Pintrich,1991) utilizado para la evaluación de las estrategias de aprendizaje y la motivación en los estudiantes. Se realizó el diseño y desarrollo de la plataforma b-learning para los estudiantes del Colegio Hacienda los Alcaparros, posteriormente se realizó la aplicación del instrumento de valoración (MSLQ) en cada uno de los grupos que hicieron parte del experimento.

El cuestionario utilizado (Motivated Strategies Learning Questionnaire (Pintrich,
1991), permtió evaluar aspectos motivacionales y cognitivos, la sección de motivación evaluó aspectos motivacionales tales como la orientación hacia metas intrínsecas, orientación hacia metas extrínsecas, valoración de la tarea, percepción de autoeficacia, control del aprendizaje y ansiedad. Por su parte, la sección relativa al uso de estrategias de aprendizaje evaluó estrategias de repaso, elaboración, organización, pensamiento crítico, autorregulación, manejo de tiempo y ambiente, regulación del esfuerzo, aprendizaje con pares y búsqueda de ayuda.

Los estudiantes encuestados que interactuaron con el ambiente b-learning obtuvieron un puntaje más alto que el grupo que continuó sus clases tradicionales, en el cuestionario MSLQ que se aplicó para evaluar la motivación y uso de estrategias de aprendizaje, los estudiantes encuestados que interactuaron con el ambiente b-learning mostraron una orientación motivacional intrínseca antes que extrínseca y presentaron niveles relativamente más altos en cuanto a las creencias de autoeficacia, en cuanto a las estrategias de aprendizaje los estudiantes tienden a utilizar estrategias de búsqueda de ayuda, aprendizaje con pares y pensamiento crítico.

Los resultados obtenidos permitieron confirmar la hipótesis planteada en este estudio: "Los estudiantes que interactúan con un ambiente b-learning presentan niveles más altos de motivación y uso de las estrategias de aprendizaje que los estudiantes de un ambiente presencial", ya que los estudiantes que interactuaron con el ambiente b-learning presentaron unos niveles más altos en cuanto a la motivación y uso de estrategias de aprendizaje. 